\title{
Erratum to: Long-term safety and efficacy of antithymocyte globulin induction: use of integrated national registry data to achieve ten-year follow-up of 10-10 Study participants
}

\author{
Krista L. Lentine ${ }^{1,2,4^{*}}$, Mark A. Schnitzler ${ }^{2}$, Huiling Xiao ${ }^{1,2}$ and Daniel C. Brennan ${ }^{3}$
}

After publication of this article, it has come to our attention that a few corrections had not been updated. We are publishing this erratum to highlight what has been updated from the original article. The updates are as follows:-

Abstract - the abstract has been updated to reflect the figures in Table 2, patient survival percentage has been updated from $52.5 \%$ to $52.8 \%$.

Table 2 - The P value for Freedom from acute rejection, graft failure or death has been updated from 0.05 to 0.04 .

Figure 3 captions were incorrect on the original article. This has now been updated. The caption for 3b was updated from Patient survival to Death-censored graft survival. The caption for $3 \mathrm{c}$ was updated from Death censored graft survival to Patient Survival.

The corresponding text relating to Fig. 3 has also been updated in line with the figure changes. In the original article it was:-

Patient survival was numerically and statistically similar in both treatment groups at 5 years and equivalent at 10 years (rATG, $52.8 \%$; basiliximab, $52.2 \%$; $P=0.92$ ) (Fig. 3b). Death-censored graft survival was also equivalent in the two groups by 10 years (rATG, $68.5 \%$; basiliximab, $68.4 \%$; two-sided $P=0.80$ ) (Fig. 3c). Combining trends in mortality and graft failure, all-cause graft survival was generally similar over time among participants randomized to both trial arms, and by 10 years was $34.3 \%$ in those treated with rATG versus $30.9 \%$ in those treated with basiliximab at (two-sided $P=0.56$ ) (Fig. 3d).
This has now been updated to the following:-

Death-censored graft survival was also equivalent in the two groups by 10 years (rATG, $68.5 \%$; basiliximab, 68.4\%; two-sided $P=0.80$ ) (Fig. 3b). Patient survival was numerically and statistically similar in both treatment groups at 5 years and equivalent at 10 years (rATG, $52.8 \%$; basiliximab, $52.2 \%$; $P=0.92$ ) (Fig. 3c). Combining trends in mortality and graft failure, all-cause graft survival was generally similar over time among participants randomized to both trial arms, and by 10 years was $34.3 \%$ in those treated with rATG versus $30.9 \%$ in those treated with basiliximab (two-sided P = 0.56) (Fig. 3d).

\section{Author details}

${ }^{1}$ Center for Outcomes Research, Saint Louis University School of Medicine, St. Louis, MO, USA. ${ }^{2}$ Abdominal Transplantation, Saint Louis University School of Medicine, St. Louis, MO, USA. ${ }^{3}$ Transplant Nephrology, Department of Medicine, Washington University School of Medicine, St. Louis, MO, USA. ${ }^{4}$ Saint Louis University, Salus Center 4th Floor, 3545 Lafayette Avenue,

St. Louis, MO 63104, USA.

Published online: 16 September 2015

Reference

1. Lentine KL, Schnitzler MA, Xiao H, Brennan DC. Long-term safety and efficacy of antithymocyte globulin induction: use of integrated national registry data to achieve ten-year follow-up of 10-10 Study participants. Trials. 2015;16:635

\footnotetext{
* Correspondence: lentinek@slu.edu

The online version of the original article can be found under doi:10.1186/s13063-015-0891-y.

${ }^{1}$ Center for Outcomes Research, Saint Louis University School of Medicine,

St. Louis, MO, USA

${ }^{2}$ Abdominal Transplantation, Saint Louis University School of Medicine,

St. Louis, MO, USA
} 